\title{
Tratamiento médico quirúrgico de una secuela de fractura mandibular infectada: reporte de caso
}

Ghersi-Miranda H, Carrión-Mauricio L. Tratamiento médico quirúrgico de una secuela de fractura mandibular infectada: reporte de caso. Rev Estomatol Herediana. 2011; 21(4):210-218.

\section{RESUMEN}

Aproximadamente 22 millones de niños sufren lesiones anualmente en el mundo, el $12 \%$ es resultado de traumas. Las fracturas mandibulares se encuentran entre ellas pudiendo, potencialmente, generar complicaciones en la erupción dental, pérdidas de dientes, alteración de la oclusión, crecimiento y desarrollo facial. Las causas frecuentes son caídas, accidentes deportivos y vehiculares. El diagnóstico es complejo, por la poca colaboración de los pacientes. Existen controversias respecto al tratamiento en pediátricos. Estas fracturas deben ser tratadas lo antes posible, teniendo en cuenta la edad, ubicación de la fractura, tiempo, etc. Se pueden presentar algunas complicaciones postoperatorias como: infecciones, alteraciones en la sensibilidad, deformidades faciales, etc. Se reporta el manejo médico quirúrgico de un paciente pediátrico, con diagnóstico de proceso infeccioso moderado agudo, asociado a secuela postquirúrgica de fractura mandibular. Fue tratado en el Servicio de Cirugía Oral y Maxilofacial de la Facultad de Estomatología de la Universidad Peruana Cayetano Heredia. Lima-Perú. Conclusiones: Las fracturas en el cuerpo mandibular rara vez influyen en el crecimiento óseo si el tratamiento es realizado rápida y correctamente. Las miniplacas reabsorbibles y metálicas son recomendables de acuerdo al operador para las fracturas maxilofaciales, no influyendo en las complicaciones postoperatorias.

Palabras clave: FRACTURAS MANDIBULARES / OSTEOMIELITIS / QUIMIOTERAPIA.

Management in mandibular fracture complication in a child. A case report ABSTRACT

Approximately 22 million children are injured each year worldwide, $12 \%$ are result of trauma. Mandibular fractures can, potentially, lead to complications in tooth eruption; tooth loss; abnormal occlusion, facial growth and development. Common causes are falls, vehicle accidents and sports. Diagnosis is complex, most of them are uncooperative patients. Some prefer the invasive management (rigid fixation) and others the conservative one. These fractures should be treated as soon as possible, considering age, fracture location, time, etc. There may be some postoperative complications as infection, hypoesthesia, facial deformities, etc. We report a surgical medical management of a pediatric patient. He came to our clinic with a diagnosis of mild acute infectious process associated with postoperative sequelae of mandibular fracture. He was treated at the Department of Oral and Maxillofacial Surgery, Faculty of Dentistry of the Universidad Peruana Cayetano Heredia. Lima-Peru. Conclusions: The mandibular body fractures rarely affect bone growth if treatment is done quickly and correctly. The reabsorbable and metal miniplates are used according to the maxillofacial surgeon, not influencing postoperative complications.

Key words: MANDIBULAR FRACTURES / OSTEOMYELITIS / DRUG THERAPY.

\section{Introducción}

Se estima que cerca de 22 millones de niños sufren lesiones anualmente en el mundo y el $12 \%$ de ellas como resultado de traumas $(1,2)$. De acuerdo a la edad la incidencia de fracturas maxilofaciales es de $1 \%$ en niños menores de 5 años y de $9 \%$ en los menores de 12 años, existiendo entonces una relación directamente proporcional a la edad (3-6).

La etiología de las fracturas faciales esta frecuentemente relacionado a violencia o actividades ocupacionales por lo que es atributo principalmente de los adultos, sin embargo en niños las injurias maxilofaciales varían ampliamente de acuerdo a factores sociales, culturales y ambientales, dentro de los que encontramos los accidentes en vehículos motorizados (5 al 80,2\%), violencia $(3,7$ al $61,1 \%)$, caídas $(7,8$ al $48 \%$ ), uso de bicicleta $(7,4$ al $48 \%$ ) y deportes (10 al 42\%) dentro de las causas más comunes (1,2,715). En cuanto al género los varones están mayormente afectados en una relación de $2: 1$ respecto a las mujeres $(1,2,6,16,17)$.

La susceptibilidad de que los traumas se localicen en la región cráneo facial en los niños se debe a que tienen mayor volumen y masa craneal con respecto al cuerpo (15). Solo del 1 al $15 \%$ de todas las fracturas faciales ocurren en la población pediátrica menor de 16 años $(2,7,8,15)$, y el $1 \%$ en aquellos menores de 5 años (1,2,10-14,18-

\section{Hugo Ghersi Miranda ${ }^{1}$ Lizeth Katheryn Carrión Mau- ricio $^{2}$}

Docente del Departamento Académico de Medicina y Cirugía Bucomaxilofacial.

${ }^{2}$ Residente del Programa de Especialización en Cirugía Oral y Maxilofacial.

Facultad de Estomatología. Universidad Peruana

Cayetano Heredia.

\section{Correspondencia}

Hugo Ghersi Miranda

Honorio Delgado 430 - Lima 31, Perú.

Teléfono: 998622777

e-mail: hugo.ghersi@upch.pe

Recibido : 11 de setiembre de 2011

Aceptado : 20 de noviembre de 2011
33).

La incidencia de fracturas faciales en pediátricos comparadas con los adultos es baja, probablemente porque el estado de maduración ósea permita que los huesos faciales sean más flexibles, además de la poca neumatización de los senos paranasales,y una cantidad importante de grasa bucal que protege la región malar en los infantes $(1,2,7,8,15)$.

Las fracturas faciales mas frecuentes en niños abarcan los huesos nasales y la mandíbula $(9,1 \%)$; el pico de incidencia de esta última se encuentra en un rango de edad entre 15 y 18 años $(1,12,17$ 20,22,27,31,32,34). Dentro de la distribución de fracturas mandibu- 
lares se describe que las condilares, subcondilares y de ángulo mandibular se presentan en un $80 \%$, las de sínfisis y parasínfisis en un 15-20\%, mientras que las de cuerpo son bastante raros (16).

Según el estudio retrospectivo de Oji (4) en 1998, donde incluyo 40 pacientes menores de 11 años, halló que 30,9\% presentaban fractura de cóndilo, $9 \%$ de cuerpo, $6 \%$ parasínfisis, $5 \%$ ángulo, $5 \%$ proceso alveolar y 4\% sínfisis. En este mismo estudio se presentaron las causas más comunes: accidente de tránsito $27.5 \%$, caídas en casa $25 \%$, accidentes de juego $20 \%$, caídas de altura $20 \%$, primeras peleas $5 \%$.

Una evaluación física minuciosa es importante porque pueden estar relacionadas con otro tipo de lesiones concomitantes. Kaban y Troulis (16) reportaron que 22 de 29 pacientes con fractura mandibular tuvieron otras lesiones que incluyeron las faciales, fractura de extremidades, cráneo, y espina cervical. Sin embargo el examen clínico y radiológico es más difícil en ellos, porque en general, los niños tienen menor masa, lo cual en episodios traumáticos resulta en una gran fuerza por unidad de área corporal, presentando menor grasa y un tejido conectivo mas elástico, estos factores resultan en múltiples lesiones de órganos internos sin presentar signos externos $(1,4,6,15)$.

Existen diferentes signos y síntomas que nos llevan a sospechar el lugar de la fractura, tales como: dolor, laceraciones, mordida abierta, retrusión mandibular, laterodesviación, trismus, alteraciones en la oclusión, equimosis ó hematomas, movilidad de segmentos óseos, alteraciones neurológicas, entre otros $(6,16)$.

Los exámenes imaginológicos para el diagnóstico incluyen: Tomo- grafía computarizada (idealmente) o radiografía panorámica, oclusal inferior, posteroanterior tipo Towne y periapicales, según el tipo de fractura que se sospeche (16).

La gran preocupación cuando se trata al paciente pediátrico es el efecto psicológico y anatómico. Durante la infancia tanto la hospitalización, el trauma y la cirugía pueden interrumpir los patrones de alimentación y sueño del niño. En la fase preescolar, la ansiedad causada por la separación de la madre, actúa como factor estresante conduciendo posiblemente a un comportamiento agresivo (1).

El manejo de las fracturas mandibulares en niños es diferente a la de adultos por las variaciones anatómicas, rápida cicatrización, grado de cooperación del paciente, y el potencial cambio en el crecimiento $(6,16,35)$. Todo esto repercute en posiciones controversiales con respecto a la decisión del tratamiento por el posible efecto en el crecimiento mandibular y movilidad de la articulación temporomandibular (ATM) (2).

Usualmente las fracturas mandibulares y alveolares en niños son manejadas de acuerdo a los principios básicos aplicados en las fracturas de adultos (17). Es importante considerar la prioridad del tratamiento de estas, para lo cual se debe tener en cuenta la edad del niño, el grado de maduración ósea, el tipo de fractura y estructuras vecinas a la zona traumatizada (2).

El tratamiento de elección depende de la edad y la etapa de desarrollo dentario. La mandíbula del niño está llena de piezas dentarias en diferentes estadios de desarrollo, y esto debe considerarse cuando se decide hacer un tratamiento cerrado con fijación intermaxilar o un tratamiento abierto con fijación interna

\section{$(1,3,16)$.}

En la planificación del tratamiento es fundamental tener en cuenta los principales puntos de crecimiento mandibular que incluyen el cartílago condilar, el borde posterior de la rama y el proceso alveolar. Estas áreas de aposición ósea permiten un incremento de la altura, longitud y ancho de la mandíbula (16).

Los dos principios a considerarse en el manejo de las fracturas mandibulares en pacientes pediátricos son: 1) corto periodo de fijación, 2) movilización temprana de la ATM en conjunto con fisioterapia. Esto se debe al alto potencial osteogénico. Siendo de dos a tres semanas el tiempo adecuado para la fijación $(1,4)$.

Kaban y Troulis (16) mencionan que los fragmentos óseos en niños se unen parcialmente a los cuatro días después de la fractura, y a los siete días se evidencia una mayor dificultad para la reducción. Por esta razón el tratamiento debe llevarse a cabo lo más pronto posible (16).

Las fracturas de cuerpo, parasínfisis y sínfisis mandibular generalmente no son desplazadas, por la elasticidad del hueso y la presencia de piezas dentarias que actúan como "goma" manteniendo los fragmentos unidos (16).

Las discrepancias oclusales leves, después de una reducción, usualmente se resuelven con la erupción de las piezas permanentes y el hueso se remodela con la función $(6,16)$.

Cuando las fracturas no están desplazadas y no producen maloclusión el manejo incluye observación, dieta blanda y evitar actividades físicas. Los controles clínicos y radiográficos serán continuos; sin embargo cuando la fractura presenta un grado mayor desplazamiento y dependiendo del mismo se optará 
por tratamientos cerrados o abiertos, de acuerdo a la edad y desarrollo dentario $(6,16)$.

En niños menores de dos años es ideal el uso de una férula lingual con fijación circunmandibular durante tres semanas. En los pacientes entre dos y seis años se puede optar por una ferulización a nivel de primeros molares, férulas o colocación de miniplacas a nivel de borde basal. Los pacientes mayores de 13 años pueden ser manejados de igual manera que los adultos porque ya presentan piezas permanentes $(1,6,16,35)$.

En los niños la cortical mandibular es más delgada que en los adultos, cercanas de ella encontramos las piezas dentarias. El trauma a los gérmenes y dientes parcialmente erupcionados puede ocurrir durante el tratamiento abierto al colocar los alambres intraóseos o tornillos de fijación de las miniplacas; lo cual repercutiría en una falta de erupción de los dientes permanentes y una atrofia de reborde alveolar $(15,16)$.

La fijación dentaria es difícil, por la morfología y el estado de reabsorción de las raíces que limita la retención de alambres y férulas (4).

La fijación es actualmente el procedimiento más utilizado dentro del manejo de las fracturas maxilofaciales, porque provee estabilidad de los fragmentos lo cual nos permite cumplir con los principios fundamentales para la reparación ósea (6,35-37). Esta técnica fue introducida hace 20 años. Fue utilizado primariamente en adultos para la restauración tridimensional de la forma y función del esqueleto facial. Actualmente se ha convertido en el estándar en el tratamiento de los pacientes con fracturas faciales. Sin embargo en niños su aplicación es aun controversial $(4,15,35)$.

Las miniplacas de titanio de bajo perfil (1,0 a 1,3mm de espesor) frecuentemente ofrecen una buena alternativa para la fijación (38).

Estudios realizados en crías de animales demostraron que la fijación con miniplacas entre las suturas craneales y faciales produjeron un retardo del crecimiento. Estos estudios demuestran que se debe ser cuidadoso en el uso de estos sistemas en niños $(15,35)$. Por otro lado también se han descrito desventajas potenciales tales como la limitación en el crecimiento, migración de las placas, reacción a cuerpo extraño, infección, extrusion y dolor en la zona de ubicación de las placas. Estas desventajas generan un gran debate sobre la seguridad y eficacia de su utilización, por lo cual se debe evaluar la posibilidad de su retiro entre 2 a 3 meses (16,34-36).

El objetivo del tratamiento debe ser la restauración tridimensional, funcional y estética. Si se requiere la fijación semirígida con miniplacas, se debe utilizar tornillos monocorticales en el borde inferior de la mandíbula con el objetivo de evitar el daño de los dientes contiguos $(15,35)$.

En la cirugía maxilofacial contemporánea, la fijación biodegradable se ha vuelto una opción para el tratamiento de la fractura y cirugía craneofacial. El rápido desarrollo de los nuevos materiales biodegradables se extiende hasta reemplazar la fijación rígida metálica (miniplacas $y$ tornillos de titanio) $(3,38)$.

Las nuevas propiedades biomecánicas de estos materiales conllevan al incremento de las indicaciones para la utilización de los mismos. Una de sus ventajas es que su reabsorción se produce al poco tiempo después de la cicatrización ósea $(3,6)$.

Eppley (35) realizó un estudio en 44 niños que presentaron fracturas maxilomandibulares con dentición decidua (primera y segunda) en un lapso de 10 años, manejándolos con fijación reabsorbible, no encontró complicaciones en ninguno de los pacientes tratados, lo que le permitió concluir que este sistema es efectivo en el manejo de las fracturas faciales de pacientes con dentición decidua.

La decisión de utilizar una fijación metálica o reabsorbible dependerá de: 1) Propiedades mecánicas: las miniplacas metálicas resisten a las fuerzas de torsión mejor que las reabsorbibles, 2) Biocompatibilidad: las reabsorbibles pueden provocar reacciones inflamatorias o a cuerpo extraño, se demostró la presencia de partículas de titanio en el sistema linfático. 3) Facilidad en manejo: el titanio es más fácil de colocar y retirar, a diferencia del sistema reabsorbible (37).

Entre las complicaciones descritas en el tratamiento de fracturas mandibulares tenemos: infecciones postoperatorias, consolidación en mala posición de los fragmentos debido al gran potencial osteogénico del niño, luxaciones dentarias, entre otras.

Las alteraciones en el crecimiento después de una fractura, especialmente de cóndilo, es una consecuencia que con frecuencia puede ser vista, aunque la compensación durante el crecimiento y desarrollo y fa función pueden contribuir en la compensación (6).

Se sabe ampliamente que todo procedimiento quirúrgico trae consigo un grado de bacteriemia y contaminación de los tejidos circundantes a la zona trabajada. En estudios relacionados se aislaron especies aerobias (Lactobacilos, Estreptococos y Estaphylococos) y anaerobias (Peptostreptococos, Eubacterium y Propionibacterium). 
El uso preventivo de antibióticos en cualquier fractura con exposición cutánea o intraoral debe considerarse como contaminada por lo cual se indicará antibióticos como tratamiento preventivo, así también cuando se realizan tratamientos invasivos $(39,40)$.

Las fracturas mandibulares son más propensas a la infección, en especial la zona de las terceras molares, por lo que en el tratamiento de estas, se deben tener algunas consideraciones que permitan controlar las complicaciones, tales como la administración de antibióticos específicos para la microflora oral, extensión de la lesión, tratamiento invasivo, tiempo quirúrgico (39).

\section{Reporte de caso}

Paciente varón de 13 años, natural y procedente de Lima-Perú acudió al Servicio de Cirugía Oral y Maxilofacial (SCOMF) de la Clínica Estomatológica Central (CEC) de la Facultad de Estomatología Roberto Beltrán (FE) de la Universidad Peruana Cayetano Heredia (UPCH), refiriendo que 18 días antes del ingreso sufre accidente de tránsito ocupante, fue trasladado a una clínica privada donde le diagnosticaron fractura mandibular motivo por el que fue intervenido
10 días después, bajo anestesia general, mediante abordaje intraoral y extraoral para la reducción y fijación con miniplacas y tornillos de titanio. Al quinto día postoperatorio presenta secreción purulenta activa por la herida intraoral asociado a trismus, edema y dolor de la zona intervenida. Es medicado con azitromicina y ciprofloxacino de $500 \mathrm{mg}$ cada uno por vía oral, sin aparente mejoría, motivo por el cual acude con sus padres a nuestro servicio para manejo especializado.

Al examen clínico extraoral presenta tumefacción a nivel de la región geniana baja con extensión a región maseterina y submaxilar izquierda, eritematoso, doloroso a la palpación, consistencia firme, con bordes poco definidos y aumento de temperatura local (Fig. 1A). En la región submaxilar se aprecia herida quirúrgica con puntos de sutura de 3 $\mathrm{cm}$ de longitud, eritematosa. Intraoralmente, presenta trismus $(10 \mathrm{~mm})$, dos heridas quirúrgicas, la primera a nivel de fondo de surco del IV cuadrante, otra en fondo de surco de III cuadrante con extensión a trígono retromolar con secreción purulenta activa. Dentición mixta, mordida abierta anterior y posterior izquierda, plano oclusal inferior alterado, movilidad de segmentos óseos entre

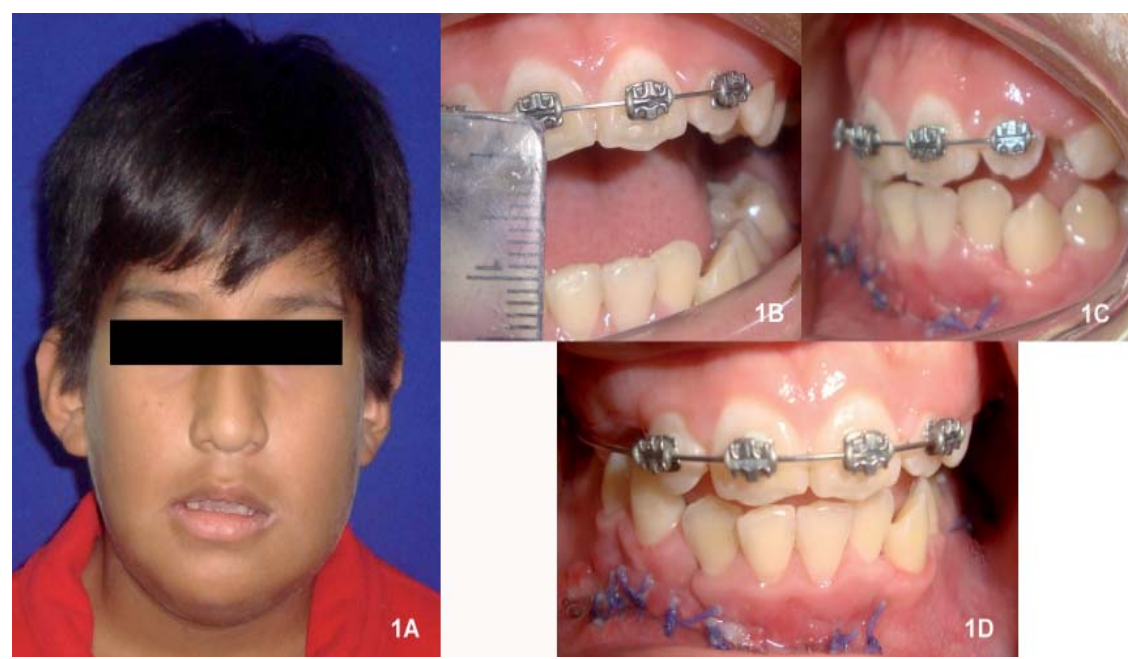

Fig. 1. Asimetría facial bilateral en región geniana baja. piezas 42 - 43 y por distal de pza 37 (Fig. 1-ABCD).

De las imágenes radiográficas (panorámica, postero-anterior tipo Towne y oclusal inferior) se puede concluir: Primer trazo de fractura que se extiende desde el reborde alveolar entre piezas 42 y 43 con trayecto oblicuo hacia distal, comprometiendo el borde basal y presencia de escalón a ese nivel. Segundo trazo de fractura por distal de la pieza 37 , abarcando la cripta del germen de la pieza 38 hasta el borde basal del ángulo mandibular imágenes radiopacas compatibles con fijación semirigida (dos miniplacas de 4 agujeros y 6 agujeros respectivamente con 4 tornillos de titanio cada una) (Fig. 2A-B).

El diagnóstico definitivo fue secuela postquirúrgica de fractura mandibular: fractura de parasínfisis derecha y fractura de ángulo mandibular izquierdo sobreinfectado.

Se inicia plan de tratamiento médico-quirúrgico de dos fases. Primera fase: tratamiento de la

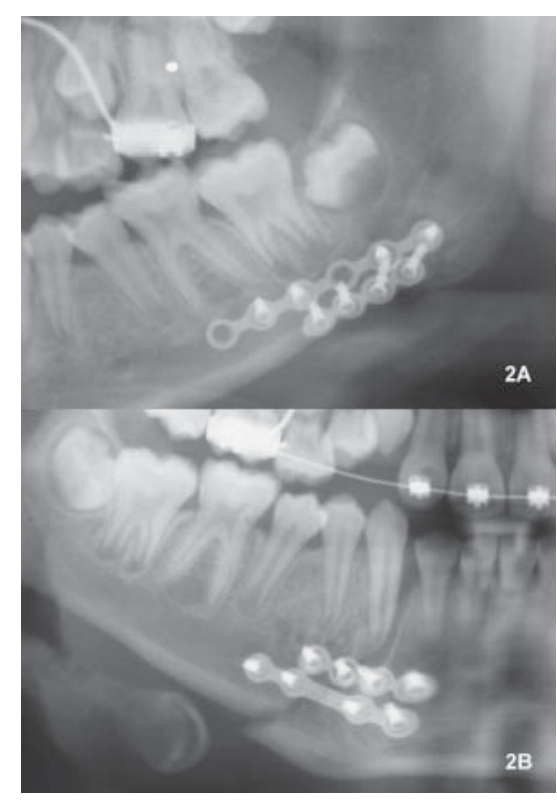

Fig. 2A.Imagenes mixtas en cuerpo mandibular a nivel de las piezas 37 y germen de 38. Presencia de dos placas de fijación. 2B. Trazo de fractura entre piezas 42 y 43 comprometiendo el borde basal. 
infección; para lo cual se suspende la antibioticoterapia previa y se inicia con clindamicina de $300 \mathrm{mg}$ c/6horas. Dos días después, se procede al drenaje a través de la herida extraoral, que permitió una mejora en los signos y síntomas (Fig. 3AB). Segunda fase: bajo anestesia general, limpieza quirúrgica, retiro de miniplacas y reposición de fragmentos óseos.

La fase quirúrgica inició con la infiltración de anestesia local a nivel trígono retromolar y fondo de surco vestibular del III y IV cuadrante y en la zona anterior. Se realizó la incisión lineal a este nivel y decolado mucoperióstico hasta evidenciar los trazos de fractura y las miniplacas de titanio, inmediatamente después se ingreso a través de la cicatriz extraoral y procedió al curetaje y lavado profuso de la zona, obteniéndose material purulento. Exodoncia del germen de la pieza 38 por estar comprometida en la línea de fractura y dentro de la zona de la infección, luego se retiraron los tornillos, miniplacas y tejido de granulación para reposicionar los fragmentos óseos previa fijación intermaxilar. Se redujo la fractura de parasínfisis según la técnica de Champi (dos miniplacas: una por debajo de los ápices radiculares y sobre el agujero mentoniano, y la segunda, $5 \mathrm{~mm}$ por encima del reborde basal). La fractura de ángulo se trató según la técnica de la AO (Asociación de Osteosíntesis), que incluye la colocación de 2 miniplacas en la cortical externa del ángulo mandibular para dar mayor fijación, ya que la infección promueve la inestabilidad de los fragmentos (Fig. 4A-B).

Los controles clínicos postoperatorios mostraron mejoría en el paciente, ausencia de secreción purulenta, apertura bucal de $40 \mathrm{~mm}$, el plano oclusal inferior adecuado y buena interdigitación entre piezas superiores e inferiores. Al examen radiográfico se evidencia trazo de fractura en parasínfisis y ángulo

mandibular derecho reducido, con material de osteosíntesis. Continuidad de rebordes basales (Fig. 5).

Se realizaron controles continuos

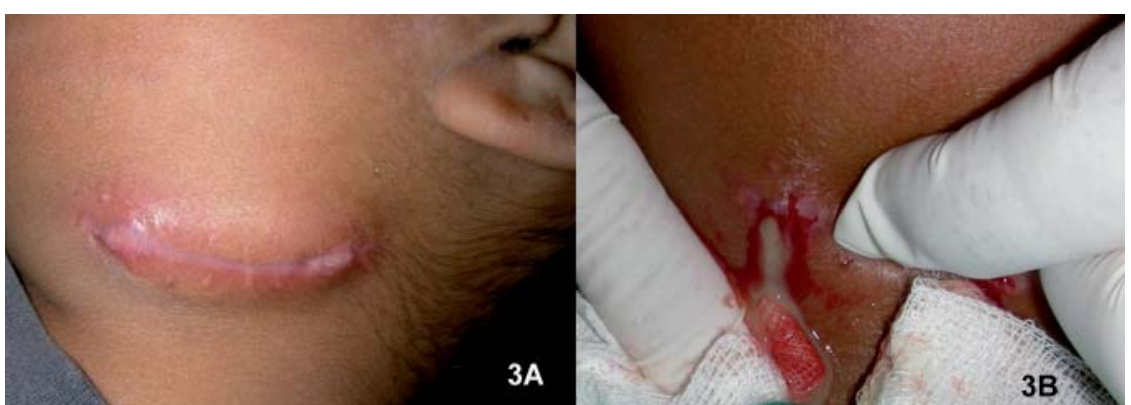

Fig. 3A. Absceso submandibular izquierdo. 3B. Drenaje del absceso submandibular.

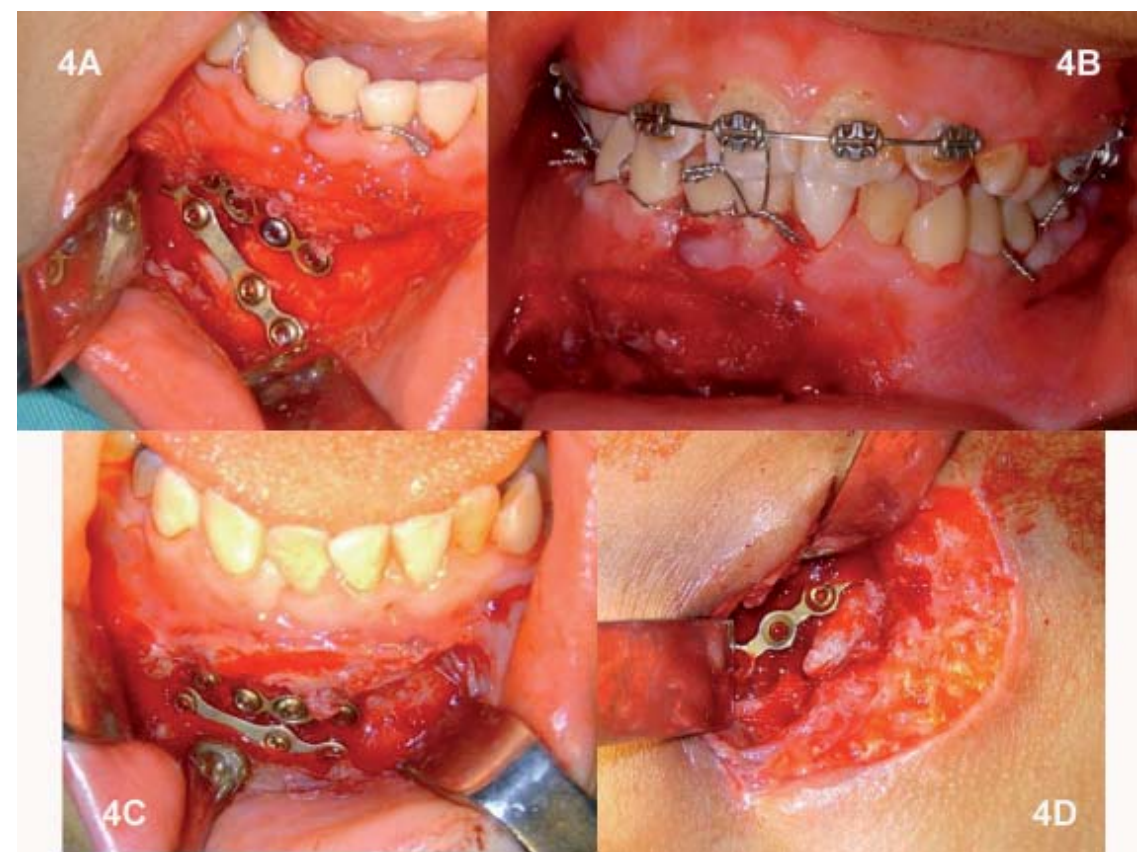

Fig. 4A. Miniplacas en el área infectada del lado derecho. 4B. Fijación maxilomandibular intraoperatoria. 4C. Reposición de miniplacas en el lado derecho, luego de limpieza quirúrgica y reducción de fractura. 4D. Fijación de fragmentos óseos del lado izquierdo con miniplaca, luego de limpieza quirúrgica y reducción de fractura. Perdida ósea en la zona.

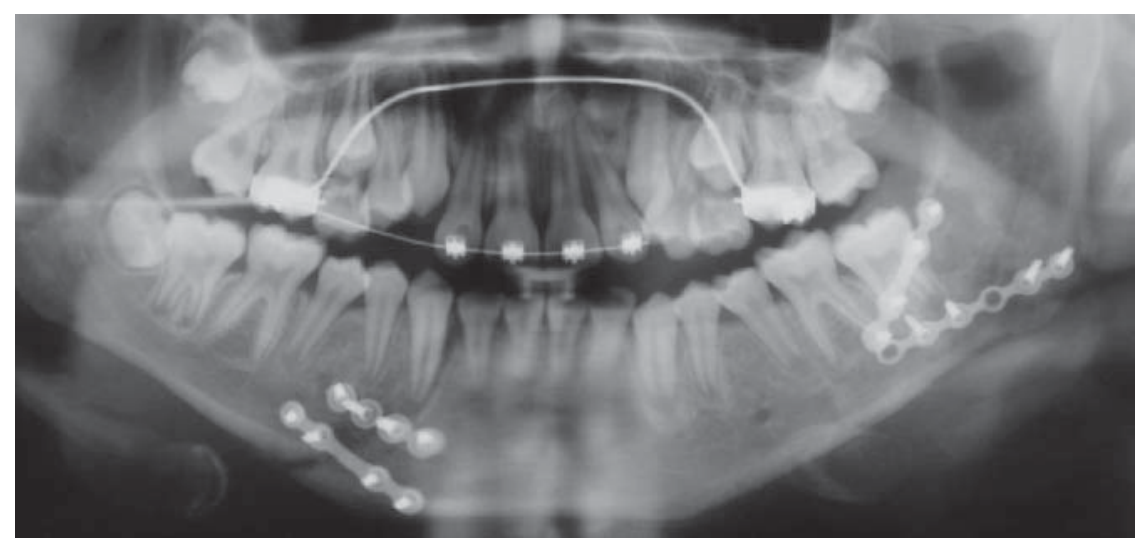

Fig. 5. XRadiografía panorámica posoperatoria. Material de osteosíntesis bilateralmente. Reducción de las fracturas. 
durante seis meses (Fig. 6A-C). En la radiografía de control, se observa continuidad ósea en las zonas de los trazos de fractura. Presencia de material de osteosíntesis. Se indica retiro de las mismas bajo anestesia general con abordaje intraoral a nivel de parasínfisis y ángulo mandibular (Fig 7 A-B).

La radiografía panorámica postinmediata de retiro de miniplacas, muestra zonas circulares radiolúcidas correspondientes a la ubicación de los tornillos de titanio. Clínicamente el paciente no presenta ninguna alteración en la oclusión (Fig 8).

\section{Discusión}

El 50\% de las fracturas en niños corresponden a fracturas mandibulares, tal como lo describen Anderson (8), Oji (4) y Eggensperger et al. (7), que además muestran como pico de incidencia los 12 años con un $74 \%$ de prevalencia en el sexo masculino. Los accidentes de tránsito, fueron la causa más frecuente, coincidiendo con nuestro caso reportado.

El crecimiento del complejo craneofacial requiere una coordinación morfogénica de todos los componentes óseos, que permita la formación tridimensional armónica de este, por lo tanto, cualquier alteración durante este proceso puede causar secuelas irreversibles. El tratamiento de las fracturas mandibulares en este grupo de pacientes es múltiple (reducciones cerradas y abiertas), tal como lo describen Kaban et al. $(16,28)$ y depende esencialmente del tipo y ubicación de la fractura, la edad y etapa de dentición del paciente. Durante el siglo pasado la utilización de miniplacas de titanio se convirtió en el estándar del tratamiento de fracturas, sin embargo en niños su utilización se vio limitada por los reportes de complicaciones

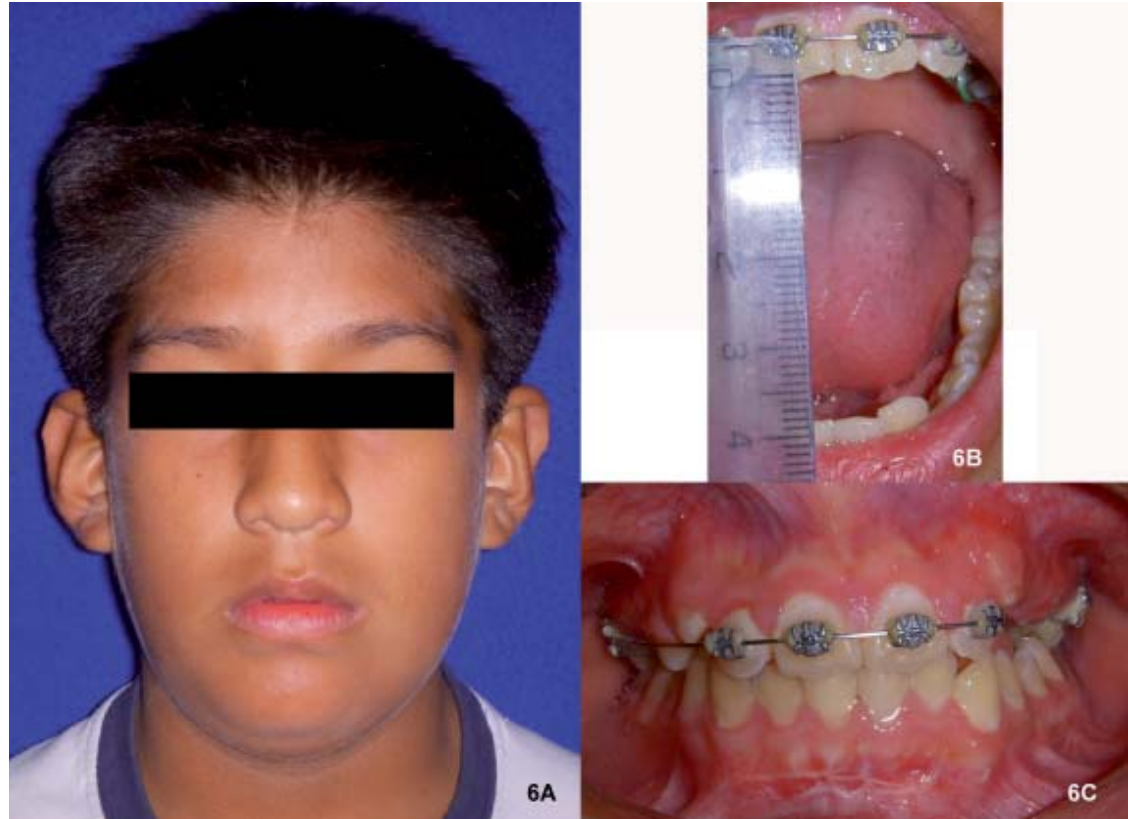

Fig. 6A. Restablecimiento de la simetría facial. 6B. Apertura bucal restablecida. 6C. Oclusión estable.

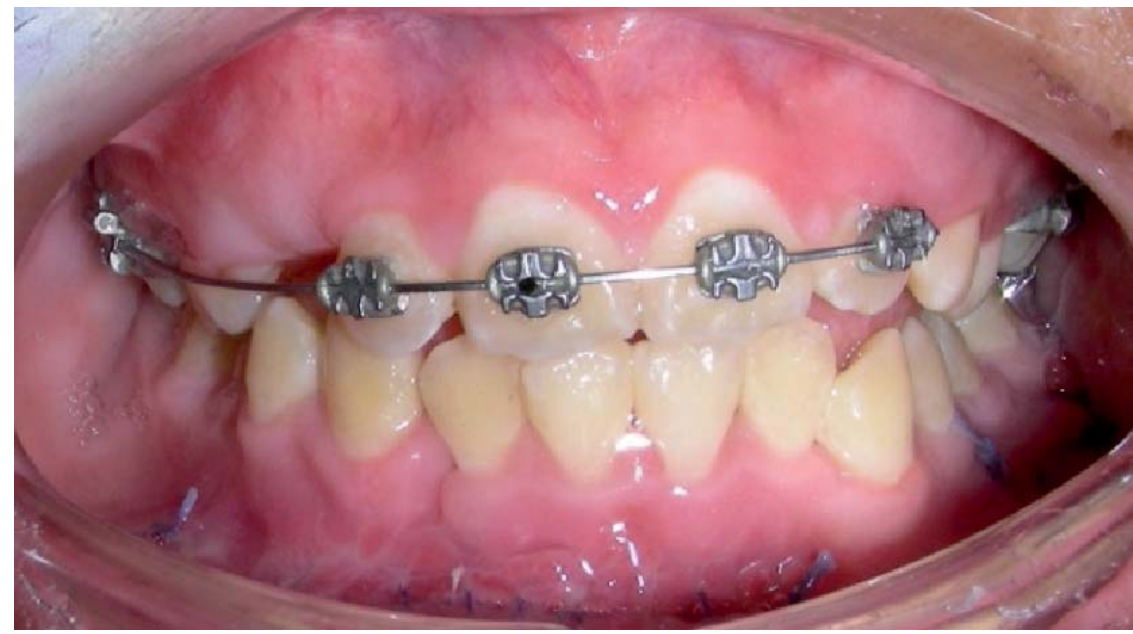

Fig. 7. Control posoperatorio a los seis meses.

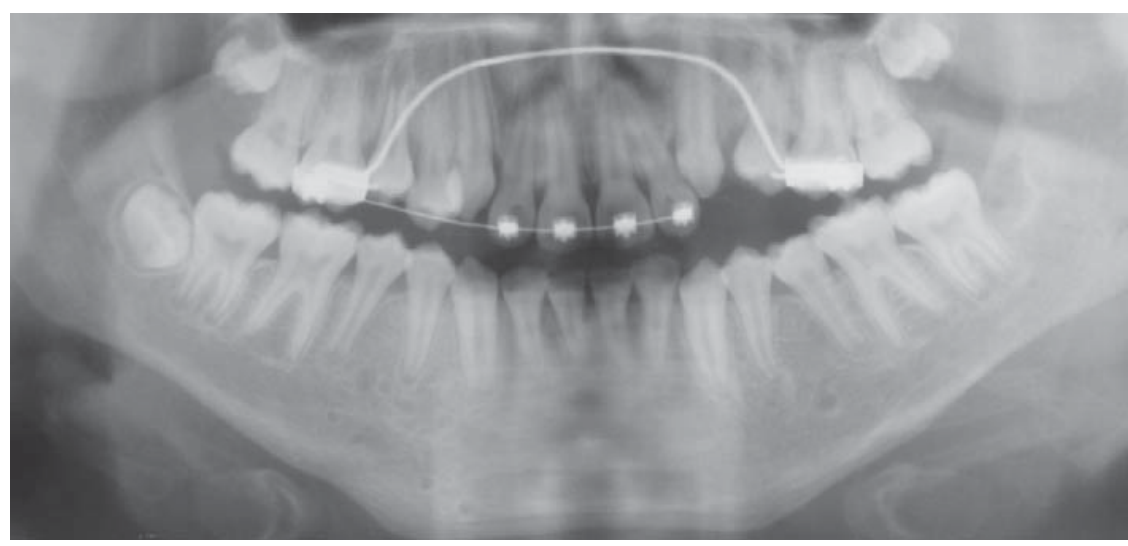

Fig. 8. Radiografía panorámica posoperatoria al retiro de miniplacas y tornillos. 
a largo tiempo. En los últimos años ha tomado auge la utilización de miniplacas reabsorbibles, desde entonces se publicaron varios estudios sobre ambas técnicas describiendo sus ventajas y desventajas.

Oji (4) estudió 40 pacientes menores de 11 años, con fracturas mandibulares a quienes trató con fijación cerrada (maxilomandibular). Un paciente presentó anquilosis temporomandibular, tres presentaron infecciones en la zona tratada y uno tuvo retardo del crecimiento mandibular por daño del centro de crecimiento condilar. Concluyó que dependiendo del caso, la reducción cerrada es una buena alternativa para el tratamiento de fracturas mandibulares en esta población.

Berryhill et al. (36), estudiaron el uso de miniplacas rígidas en niños con edad promedio de 3,9 años; incluyendo un total de 121 procedimientos y 5 años de seguimiento. Se colocaron 375 miniplacas de titanio y 1944 tornillos. El 15\% de los pacientes presentaron complicaciones dentro de las que se hallaron: cinco casos de restricción de crecimiento, cuatro presentaron migración de la placa (ninguna intracraneal), nueve casos eran palpables, tres presentaron secreciones, dos meningitis y en ocho casos se retiraron las miniplacas por las causas mencionadas. Concluyeron que pese a que existe riesgo de secuelas postoperatorias, estas son poco comunes y solo en algunos casos se retiraron las miniplacas. Yerit et al. (3), estudiaron la estabilidad y eficiencia de las miniplacas y tornillos reabsorbibles en la fijación interna en una población de 13 pacientes pediátricos con fracturas de parasínfisis, ángulo, cóndilo y combinación de las mismas. Fueron seguidos hasta 26 meses. Concluyeron que este material de osteosíntesis dio muy buena fijación y estabilidad, además las fracturas cicatrizaron sin complicaciones. Sin embargo podrían presentarse problemas potenciales relacionados con la reabsorción y crecimiento óseo, por lo que deben ser observados y estudiados a largo tiempo.

Esen et al. (38), compararon la estabilidad entre las miniplacas reabsorbibles y los sistemas de fijación metálica para el tratamiento de fracturas mandibulares, donde incluyeron 21 hemimandibulas de oveja separados en tres grupos: 1) fijación con una miniplaca de titanio 2) fijación con una miniplaca reabsorbible 3) fijación con 2 miniplacas reabsorbibles, a cada grupo se aplicaron fuerzas desde 10 a 100 Newtons (N). Demostraron que la miniplaca de titanio presenta una mayor resistencia a las cargas oclusales que el sistema reabsorbible, sin embargo la colocación de una segunda placa reabsorbible aumenta favorablemente el comportamiento biomecánico en el lugar de la fractura.

La ocurrencia de infecciones pre y/o postoperatorias en el tratamiento de fracturas mandibulares continua siendo uno de los puntos más importantes dentro de la cirugía maxilofacial, porque de ello depende la rápida recuperación y éxito del tratamiento, además de la disminución de secuelas (mala unión, osteomielitis, deformidades esqueléticas, maloclusiones, etc), evitando más intervenciones quirúrgicas. Andreasen et al. (39) hicieron un meta análisis respecto al posible beneficio de terapia antibiótica en el manejo de las fracturas mandibulares, concluyeron que la administración antibiótica por tiempo corto (Una dosis o hasta 48 horas) disminuye el número de infecciones postoperatorias. El caso que reportamos presentó una infección postoperatoria inmediata después de la primera cirugía a la que fue sometido el paciente, este repercutió en sus funciones, estado físico y psicológico, los que complican el retratamiento del mismo.

En nuestro paciente se usaron miniplacas de titanio para la fijación de los fragmentos óseos, dándonos buenos resultados, tanto funcionales como estéticos. Sin embargo, tomando en cuenta que aun se encontraba en etapa de crecimiento, se decidió esperar seis meses para la consolidación total de la fractura; luego de la cual se procedió con el retiro de las miniplacas, obteniendo resultados favorables disminuyendo las posibles secuelas.

\section{Conclusiones}

- Las fracturas de huesos faciales en niños son poco comunes y más aun las que se originan en accidentes por motociclismo.

- Las fracturas en el cuerpo mandibular rara vez influyen en el crecimiento óseo si el tratamiento es realizado rápida y correctamente.

- La osteosíntesis reabsorbibles y metálicas permiten mantener los fragmentos inmovilizados. No influyendo en las complicaciones postoperatorias.

- El manejo de las patologías traumáticas, debe ser realizado por especialistas que conozca de oclusión dentaria, microflora oral y el sistema estomatognático, para que se restituya la función, estética y el crecimiento y desarrollo pertinentemente.

\section{Referencias bibliográficas}

1. Haug RH, Foss J. Maxillofacial injuries in the pediatric patient. Oral Surg Oral Med Oral Pathol Oral Radiol Endod. 2000; 90(2):126-34. 
2. Rahman RA, Ramli R, Rahman NA, Hussaini HM, Idrus SM, Hamid AL. Maxillofacial trauma of pediatric patients in Malaysia: a retrospective study from 1999 to 2001 in three hospitals. Int J Pediatr Otorhinolaryngol. 2007; 71(6):929-36.

3. Yerit KC, Hainich S, Enislidis G, Turhani D, Klug C, Wittwer G, Ockher M, Undt G, Kermer C, Watzinger F, Ewers R. Biodegradable fixation of mandibular fractures in children: stability and early results. Oral Surg Oral Med Oral Pathol Oral Radiol Endod. 2005; 100(1):17-24.

4. Oji C. Fractures of the facial skeleton in children: a survey of patients under the age of 11 years. J Craniomaxillofac Surg. 1998; 26(5):322-5.

5. Tuovinen V, van Steenis K, Sindet-Pedersen S. Internal fixation of a mandibular fracture in a 6-month-old girl--a case report. Int J Oral Maxillofac Surg. 1995; 24(3):210-1.

6. Zimmermann CE, Troulis MJ, Kaban LB. Pediatric facial fractures: recent advances in prevention, diagnosis and management. Int J Oral Maxillofac Surg. 2005; 34(8):823-33.

7. Eggensperger Wymann NM, Hölzle A, Zachariou Z, Iizuka T. Pediatric craniofacial trauma. J Oral Maxillofac Surg. 2008; 66(1):58-64.

8. Anderson PJ. Fractures of the facial skeleton in children. Injury. 1995; 26(1):47-50.

9. Chidzonga MM. Facial fractures in children. Cent Afr J Med. 1987; 33(12):274-7.

10. Iizuka T, Thorén H, Annino DJ Jr, Hallikainen D, Lindqvist C. Midfacial fractures in pediatric patients. Frequency, characteristics, and causes. Arch Oto- laryngol Head Neck Surg. 1995; 121(12):1366-71.

11. Stylogianni L, Arsenopoulos A, Patrikiou A. Fractures of the facial skeleton in children. $\mathrm{Br}$ J Oral Maxillofac Surg. 1991; 29(1):9-11.

12. Güven O. Fractures of the maxillofacial region in children. $\mathrm{J}$ Craniomaxillofac Surg. 1992; 20(6):244-7.

13. Thorén H, Iizuka T, Hallikainen D, Lindqvist C. Different patterns of mandibular fractures in children. An analysis of 220 fractures in 157 patients. $J$ Craniomaxillofac Surg. 1992; 20(7):292-6.

14. Kountakis SE, Rafie JJ, Ghorayeb B, Stiernberg CM. Pediatric gunshot wounds to the head and neck. Otolaryngol Head Neck Surg. 1996; 114(6):756-60.

15. Abdullah WA. The use of a single titanium microplate in displaced pediatric parasymphysial mandibular fractures. Saudi Dent J. 2009; 21(2):95-100.

16.Kaban L, Troulis M. Facial Trauma II: dentoalveolar injuries and mandibular fractures. En: Pediatric Oral and Maxillofacial Surgery. Pensylvania: Saunders; 2004. 441-61.

17.Rémi M, Christine MC, Gael P, Soizick P, Joseph-André J. Mandibular fractures in children: long term results. Int $\mathrm{J}$ Pediatr Otorhinolaryngol. 2003; 67(1):25-30.

18. Gussack GS, Luterman A, Powell RW, Rodgers K, Ramenofsky ML. Pediatric maxillofacial trauma: unique features in diagnosis and treatment. Laryngoscope. 1987; 97(8 Pt 1):925-30.

19.Adekeye EO. Pediatric fractures of the facial skeleton: a survey of 85 cases from Kaduna, Nigeria. J Oral Surg. 1980; 38(5):355-8.
20. Sherick DG, Buchman SR, Patel PP. Pediatric facial fractures: analysis of differences in subspecialty care. Plast Reconstr Surg. 1998; 102(1):28-31.

21.Zachariades N, Papavassiliou D, Koumoura F. Fractures of the facial skeleton in children. J Craniomaxillofac Surg. 1990; 18(4):151-3.

22.McGraw BL, Cole RR. Pediatric maxillofacial trauma. Agerelated variations in injury. Arch Otolaryngol Head Neck Surg. 1990; 116(1):41-5.

23.Koltai PJ, Amjad I, Meyer D, Feustel PJ. Orbital fractures in children. Arch Otolaryngol Head Neck Surg. 1995; 121(12):13759.

24.Thorén H, Iizuka T, Hallikainen $D$, Nurminen $M$, Lindqvist $C$. An epidemiological study of patterns of condylar fractures in children. Br J Oral Maxillofac Surg. 1997; 35(5):306-11.

25.Thaller SR, Huang V. Midfacial fractures in the pediatric population. Ann Plast Surg. 1992; 29(4):348-52.

26.Posnick JC, Wells M, Pron GE. Pediatric facial fractures: evolving patterns of treatment. J Oral Maxillofac Surg. 1993; 51(8):836-44.

27.Fortunato MA, Fielding AF, Guernsey LH. Facial bone fractures in children. Oral Surg Oral Med Oral Pathol. 1982; 53(3):225-30.

28.Kaban LB, Mulliken JB, Murray JE. Facial fractures in children: an analysis of 122 fractures in 109 patients. Plast Reconstr Surg. 1977; 59(1):15-20.

29. Tanaka N, Uchide N, Suzuki $\mathrm{K}$, Tashiro T, Tomitsuka K, Kimijima Y, Amagasa T. Maxi1lofacial fractures in children. $\mathrm{J}$ Craniomaxillofac Surg. 1993; 
21(7):289-93.

30.Demas PN, Braun TW. Pediatric facial injuries associated with all-terrain vehicles. J Oral Maxillofac Surg. 1992; 50(12):12803.

31.Hill CM, Crosher RF, Mason DA. Dental and facial injuries following sports accidents: a study of 130 patients. Br J Oral Maxillofac Surg. 1985; 23(4):268-74.

32. McGraw-Wall BL. Facial fractures in children. Facial Plast Surg. 1990; 7(3):198-205.

33. Ramba J. Fractures of facial bones in children. Int J Oral Surg. 1985; 14(6):472-8.

34.Lamphier J, Ziccardi V, Ruvo
A, Janel M. Complications of mandibular fractures in an urban teaching center. J Oral Maxillofac Surg. 2003;61(7):745-9.

35.Eppley BL. Use of resorbable plates and screws in pediatric facial fractures. J Oral Maxillofac Surg. 2005; 63(3):385-91.

36. Berryhill WE, Rimell FL, Ness J, Marentette L, Haines SJ. Fate of rigid fixation in pediatric craniofacial surgery. Otolaryngol Head Neck Surg. 1999; 121(3):26973.

37.Bos RR. Treatment of pediatric facial fractures: the case for metallic fixation. J Oral Maxillofac Surg. 2005; 63(3):382-4.

38.Esen A, Ataoğlu H, Gemi L.
Comparison of stability of titanium and absorbable plate and screw fixation for mandibular angle fractures. Oral Surg Oral Med Oral Pathol Oral Radiol Endod. 2008; 106(6):806-11.

39.Andreasen JO, Jensen SS, Schwartz O, Hillerup Y. A systematic review of prophylactic antibiotics in the surgical treatment of maxillofacial fractures. J Oral Maxillofac Surg. 2006; 64(11):1664-8.

40. Rajasuo A, Nyfors S, Kanervo A, Jousimies-Somer H, Lindqvist C, Suuronen R. Bacteremia after plate removal and tooth extraction. Int J Oral Maxillofac Surg. 2004; 33(4):356-60. 